\title{
1 - Química Organometálica: Os meus últimos 30 anos
}

0 S VENTOS DE AGGIORNAMENTO CIENTIFICO que começaram a soprar no IST, de forma tímida mas consistente, nos finais dos anos sessenta, trouxeram consigo uns tantos "conferencistas estrangeiros", personagens mitificadas pelo nosso isolamento no mundo e pela falta de congéneres autóctones. A minha curiosidade estudantil, que à data se dividia entre as múltiplas vertentes miríficas que esses tempos ofereciam, levou-me também a escutar a "mensagem" de que eram portadores. Na polivalente "Sala 24" do Departamento de Engenharia Química do IST fiquei de olhos em alvo perante a "revelação" trazida pelo Professor R. B. Gillard numa conferência, para mim inesquecível, sobre Química de Coordenação. Inesquecivel não porque me recorde sequer do tema específico mas porque marcou definitivamente o meu interesse pela Química dos Metais de Transição como tema central de uma carreira académica e de investigação que, entretanto, já decidira abraçar. A primeira oportunidade surgiu em Setembro/Outubro de 1970 ao realizar um estágio com o Prof. A. Romão Dias, recentemente doutorado na Universidade de Oxford. A sua área de trabalho, Química Organometálica, conciliava de modo particularmente feliz o meu interesse pela sintese orgânica com o novo arrebatamento pela química dos metais. 0 trabalho resumiu-se a pouco mais do que tentar erguer um laboratório adequado, mas foi definitivamente convincente. 0 estágio final de curso realizado no Instituto de Química Orgânica-TNO, Utrecht, (Agos-
to/Outubro 1971), instituição dirigida por um dos fundadores da Quimica Organometálica, Prof. van der Kerk, valeu-me o primeiro artigo de investigação em Química Organometálica de Sn e Sb.

No regresso, como Assitente Estagiário de Química Inorgânica, integrei definitivamente o grupo do Prof. Romão Dias começando a trabalhar na química dos metalocenos de Mo e W que ele havia herdado do seu Doutoramento em Oxford e que eu, em boa verdade, quase nunca mais abandonei.

Segundo o meu caderno de laboratório desse tempo, em 6 de Dezembro de 1971 arranquei com a minha primeira "Big Prep", nome de código para a síntese dos hidretos $\left(\mathrm{C}_{5} \mathrm{H}_{5}\right)_{2} \mathrm{MH}_{2}(\mathrm{M}=\mathrm{Mo}$, W) em escala verdadeiramente imponente e marcante para qualquer principiante. Partindo destes compostos abordei a sintese de complexos de coordenação com os ligandos organometálicos de fórmula geral $\mathrm{Cp}_{2} \mathrm{M}(\mathrm{SR})_{2}(\mathrm{Cp}=$ $\mathrm{C}_{5} \mathrm{H}_{5} ; \mathrm{M}=\mathrm{Mo}, \mathrm{W}$ ). Este tema havia sido iniciado pelo Prof. Romão Dias durante a sua Tese de Doutoramento com o Prof. M. L. H. Green, e prenunciava um novo tipo de complexos polinucleares

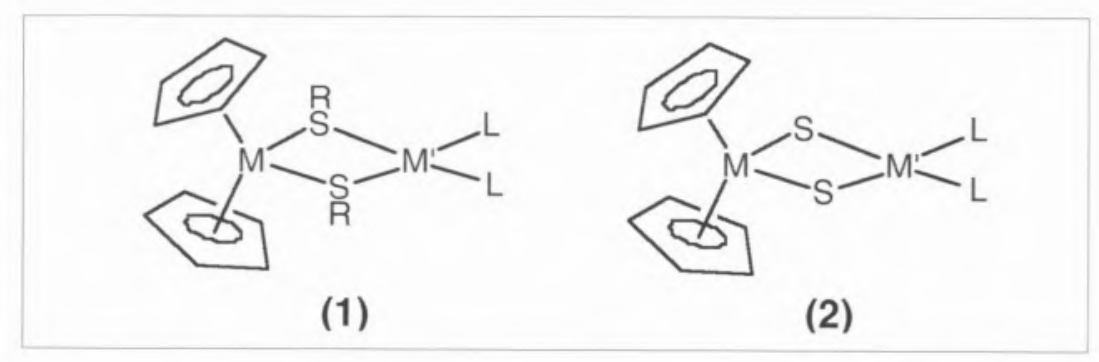

cujas múltiplas funcionalidades deixavam entrever a existência de interessantes propriedades. De facto, os complexos preparados pelo Prof. Romão Dias foram dos primeiros exemplos de espécies polifuncionais apresentando simultâneamente um ambiente organometálico em torno do $\mathrm{Mo}(\mathrm{W})$ e outro tipicamente inorgânico, de coordenação clássica, em torno de um segundo elemento metálico, como no exemplo (1).

A partida do Prof. Romão Dias para o serviço militar em Angola interrompeu este trabalho mas permitiu-me, a seu conselho, seguir para o laboratório do Prof. Green, com o objectivo de sintetizar os compostos análogos (2) com pontes de $\mathrm{S}^{2-}$ em vez das pontes de tiolato, SR (1973/74). Os primeiros 6 meses em Oxford resultaram em muitos pós pretos mas nenhum composto definido. Deste negrume, salvou-me a mudança de tema: troquei os compostos polinucleares por estudos de reactividade de compostos organometálicos, nomeadamente em ataques nucleófilos sobre espécies catiónicas de 18 electrões. Este novo tema acabou por dominar a minha Tese de Doutoramento que continuei a realizar em Portugal já sob a

\footnotetext{
*Instituto de Tecnologia Quimica e Biológica, Quinta do Marquês, Apt. 127, 2781-901 Oeiras. ccr@itqb.unl.pt
} 
orientação do Prof. Romão Dias entretanto regressado de Angola. Além do meu supervisor, os tempos auspiciosos que então se viviam trouxeram ainda a esperança de melhores tempos e, também, alguns vislumbres de que essas esperanças se poderiam concretizar. Entre estes, o maior, para a minha geração no IST, foi o início de funcionamento do Complexo Interdisciplinar. Havia finalmente instalações condignas para trabalhar em investigação, num edifício que abrigava variadas linhas de investigação e onde o entusiasmo dos "neófitos" como eu, e a determinação dos respectivos supervisores, permitiram uma vivência altamente criativa e uma progressão sustentada que acabou por ser marcante no panorama científico português.

Foi neste ambiente que me doutorei em 1978, após ter sintetizado e caracterizado numerosos derivados catiónicos e neutros de $C p_{2} \mathrm{M}$, e estudado algumas das suas reacções de ataque nucleófilo. (Esquema 1)

No essencial, foi uma tese de síntese organometálica e de desenvolvimento das respectivas metodologias, tentando estabelecer o quadro de regras ou condições que permitam prever o decorrer de reacçōes organometálicas. Este tipo de sistematização de metodologias de síntese organometálica constituiu para mim uma constante preocupação que vim posteriormente a alargar a outras áreas do meu trabalho.

Por essa altura, o grupo do Prof. Romão Dias tinha crescido de forma diversificada permitindo a abordagem de alguns problemas de uma forma conjunta e apoiada em diversos tipos de metodologias. Contudo, a Catálise, tema de aplicação por excelência da Química Organometálica, continuava por implementar. Juntando Catálise com temas da moda, parti para o Max-Planck Institut für Kohlenforschung, em Mülheim/Ruhr, Alemanha, para realizar um pós-doutoramento em catálise de activação de $\mathrm{CO}_{2}$, sob a orientação do Prof. P. W. Jolly. As voltas do destino inviabilizaram este trabalho mas abriram-me as portas para outra área intimamente relacionada com a Catálise, e que era o ex-libris do referido MPI: a química dos complexos alílicos. Nesses idos de 1982/84, tal como antes em Oxford, os 6 primeiros meses foram bem escurinhos mas os resultados posteriores abriram um pequeno capítulo na Química Organometálica do molibdénio, posteriormente estendido por Jolly e colaboradores ao W e ao Cr. [1] A actividade catalítica destes complexos na polimerização de etileno e butadieno só era superada pela sua instabilidade térmica, o que se traduziu em 18 meses de suor na mais proficua e intensa experiência de síntese organometálica da minha vida. Infelizmente, foi precisamente essa instabilidade térmica que veio a bloquear a continuação dessa linha de investigação quando, de volta ao IST, comecei a orientar e co-orientar Teses de Mestrado (José Cardoso de Menezes, Luis Veiros) e Doutoramento (Cristina Azevedo, Ana Margarida Martins, Pedro Teixeira Gomes). Nunca ninguém sob a minha orientação trabalhou com compostos tão radicalmente instáveis (a tudo!) como os dois primeiros: se a isso se deve o seu posterior abandono de uma carreira na
Química de Síntese, aqui fica o meu público pedido de desculpas! Aos outros jovens cientistas desta segunda geração de "Químicos do Complexo" valeu, para além da competência, o entusiasmo e a criatividade para ultrapassarem as dificuldades e fazer progredir a Síntese e a Catálise. À Cristina Azevedo coube alargar a química dos alilos de molibdénio alicerçada na experiência que obteve no seu Mestrado, executado no MPI de MüIheim, e iniciar a exploração da química dos metalocenos $\mathrm{Cp}_{2} \mathrm{MoL}$ ( $\mathrm{L} \neq \mathrm{CO}$, alceno, alcino) até aí inexplorada por falta de vias de acesso sintético adequadas.[2] À Ana Margarida Martins coube o olfacticamente penoso estudo da coordenação e activação de isonitrilos com o fragmento $\mathrm{Cp}_{2}$ Mo.[3] Estes trabalhos marcaram o meu regresso à química dos metalocenos tendo por fim explorar as suas evidentes capacidades de activação de ligandos, nomeadamente as que são oferecidas pelas espécies catiónicas contendo ligandos insaturados, $\left[\mathrm{Cp}_{2} \mathrm{ML}_{2}\right]^{2+}$ $(\mathrm{L}=\mathrm{CNR}, \mathrm{CO})$ tentando completar o quadro aberto muito tempo antes por M. L. H. Green e colaboradores no que respeita aos casos com $\mathrm{L}=$ alceno.

Com o trabalho de Pedro T. Gomes, entrou-se finalmente no estudo da Catálise de Polimerização Homogénea. Explorando a reactividade de complexos alílicos catiónicos de $\mathrm{Ni}(\mathrm{II}),\left[\left(\eta^{3}\right.\right.$-alilo) $\left.\mathrm{Ni}\left(\mathrm{PR}_{3}\right)_{2}\right]^{+}$, que havia preparado durante o seu Mestrado em Toulouse sob a orientação do Dr. I. Tkatchenko, obteve um notável conjunto de resultados que Ihe permitiram estabelecer definitivamente a área da Catálise de Polimerização que actualmente prossegue no

esquema 1: Ataque sequencial e estereoespecifico de $\mathrm{H}^{-}$(D) em hidrocarbonetos coordenados.

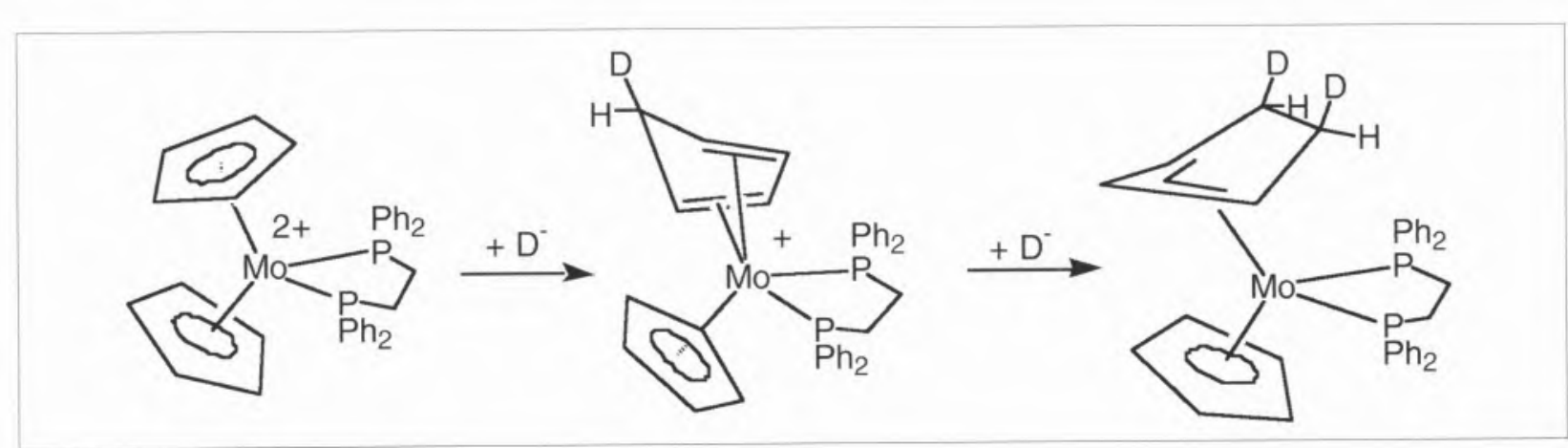




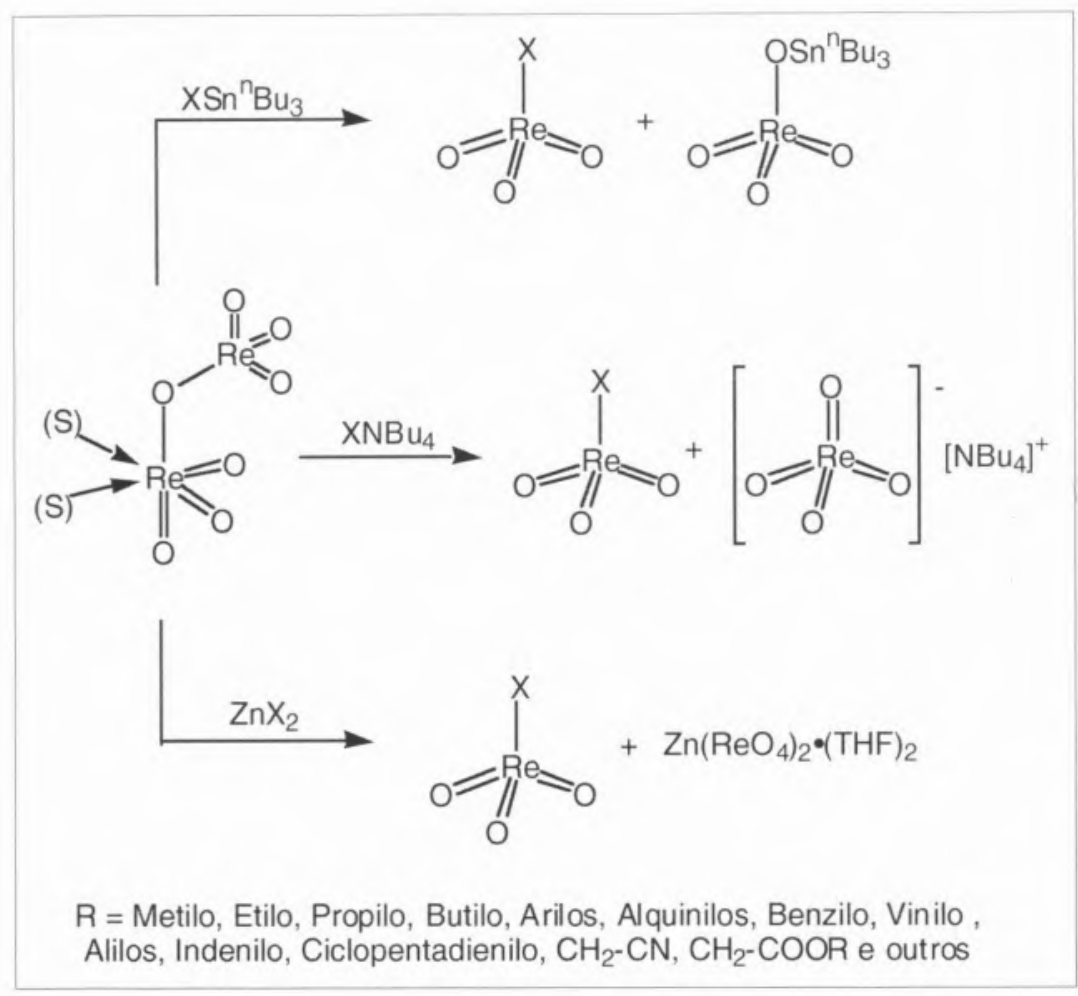

esquema 2 : Vias de síntese para derivados do tipo $\mathrm{RReO}_{3}$.

Complexo, entretanto rebaptizado de Centro de Química Estrutural algures durante o desenrolar desta narrativa. Acresce ainda distinguir a contribuição insubstituivel do Prof. José Ascenso neste esforço, ao implementar as técnicas de análise de micro-estruturas poliméricas por RMN.[4] A maturidade do grupo era agora notável. Talvez o exemplo mais acabado dessa maturidade ciientífica tenha sido 0 artigo que marcou a entrada do grupo no Organometallics com um trabalho abrangente de síntese, electroquímica, cristalografia, termoquímica e cálculos teóricos, aplicado ao estudo da eliminação $B$ em derivados do tipo $\mathrm{Cp}_{2} \mathrm{MR}_{2}\left(\mathrm{R}=\mathrm{C}_{2} \mathrm{H}_{5}, \mathrm{n}-\mathrm{C}_{4} \mathrm{H}_{9}\right)$. [5]

Foi durante a fase final destes trabalhos que me surgiu o convite do Prof. António Xavier para integrar o Centro de Tecnologia Química e Biológica, em Oeiras, (actualmente Instituto de Tecnologia Química e Biológica) como responsável por uma linha de investigação em Química Inorgânica/Organometálica/Catálise, convite esse que muito me honrou e prontamente aceitei. Ao estabelecer os objectivos a atingir no âmbito do quadro de investigação de uma instituição desta natureza, a minha opção foi pelo arranque de linhas de trabalho orientadas para intervir a médio prazo nas áreas de Sintese de Moléculas de Interesse Biológico. Dentro destas linhas, a Catálise impunha-se como área central pois a Química Organometálica ganhava uma dimensão cada vez mais forte como ferramenta de Síntese Orgânica agora já estendida a domínios claramente fora do âmbito dos processos ligados à história do seu crescimento em torno da indústria petroquímica (polimerização, hidroformilação, etc.). A Catálise Assimétrica tornava-se prioritária e exigia a sintese de complexos contendo centros estereogénicos bem definidos, estáveis e capazes de induzir reacções assimétricas na sua esfera de coordenação. A oxidação de substratos orgânicos, nomeadamente olefinas, tioéteres, alcoóis, aldeídos e outros, quer na sua vertente simétrica quer assimétrica, ganhava crescente importância na sintese de moléculas de interesse farmacológico e nos processos de descontaminação ambiental quer por oxidação de resíduos quer pela substitutição de agentes oxidantes tradicionais altamente poluentes.
Fora do âmbito da Catálise, os complexos polinucleares (re)surgiam como tema de grande actualidade quer como modelos de sistemas bio-inorgânicos, quer como sensores moleculares capazes de intervir, de forma selectiva, na monitorização e controle de processos de relevância biológica ou outra.

Com base neste quadro centrei a actividade de investigação do meu grupo em três áreas principais: catálise de oxidaçẫo com óxidos inorgânicos e organometálicos; complexos organometálicos polinucleares; complexos estruturalmente flexíveis.

De uma variedade exótica de compostos organometálicos em altos estados de oxidação, os óxidos organometálicos tinham-se tornado, em 1990, num fértil campo de investigação donde espreitavam inesperadas oportunidades em termos de Catálise. Como esse trabalho tinha a sua máxima expressão no grupo do Prof. W. A. Herrmann, da Universidade Técnica de Munique (UTM) foi para lá que me dirigi em Licença Sabática de 6 meses. Neste regresso activo à bancada o meu interesse centrou-se no desenvolvimento de métodos de síntese fiáveis e sistemáticos de óxidos organometálicos de rénio cuja sintese "ia dando" conforme a sorte ditava. Desse trabalho e do que se seguiu em visitas posteriores, resultaram métodos sintéticos gerais para compostos do tipo $\mathrm{RReO}_{3}$ com uma total amplitude da natureza de $R$, que inclui alquilos e arilos simples e funcionalizados (Esquema 2).[6] Estes estudos permitiram varrer 0 potencial da Química destes sistemas com aplicaçōes desde a Catálise aos Novos Materiais.[7]

Do rénio passou-se ao molibdénio e, mais recentemente, ao tungsténio preparando e estudando a "performance" catalítica de uma larga família de oxo-complexos organometálicos e inorgânicos na epoxidação de alcenos [8] e noutras reacções catalíticas, como a reacção de Bayer-Villiger e a olefinação de aldeídos, versão catalítica da reacção de Wittig.

Este trabalho alicerçou-se nas Teses de Doutoramento de André Lopes (ITQB) e de Ana Santos (UTM), e continua hoje 


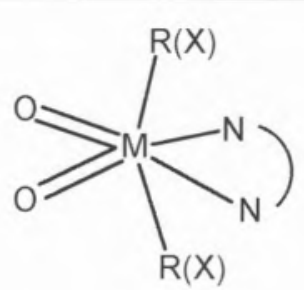

$\overbrace{\mathrm{N}}=\alpha$-diiminas, polipirazolilos, amino-alcoóis

$\mathrm{X}=\mathrm{Cl}, \mathrm{Br} ; \mathrm{R}=\mathrm{Me}, \mathrm{Et}$

esquema 3: Fórmula geral dos catalisadores de oxidaçāo de Mo e W.

em colaboração estreita com o grupo do Prof. W. Herrmann e Priv. Doz. Dr. Fritz Kühn, da UTM, onde exerci funções de orientação científica nesta área, com a Universidade de Aveiro (João Rocha e Isabel Gonçalves) e com a Universidade do Algarve (André Lopes) tendo como linha de força mais actual a síntese de catalisadores de oxidação enantioselectivos.

No campo dos complexos polinucleares o trabalho, essencialmente realizado por André Lopes e Isabel Gonçalves, centrou-se sobre espécies contendo metais em estados de oxidação díspares (um alto e um baixo) sendo que, na maior parte desses sistemas se ponteavam ambientes de coordenação organometálicos com ambientes de coordenação inorgânicos como em (3). [9]

Este trabalho teve múltiplas colaborações, incluindo as teóricas (Maria José Calhorda) as fotoquímicas (Fernando Pina, CQFB/UNL) e magnetoquímicas (Rui Teives Henriques, IST) e mantém-se vivo na busca de novos tipos de ligandos organometálicos.

A abordagem ao problema dos complexos estruturalmente flexíveis baseou-se na síntese de derivados modificados do molibdenoceno e tungstenoceno: $\mathrm{CpCp} \mathrm{MXY}^{\prime}\left(\mathrm{M}=\mathrm{Mo}, \mathrm{W} ; \mathrm{Cp} \mathrm{p}^{\prime}=\mathrm{C}_{5} \mathrm{H}_{5}\right.$, $\mathrm{C}_{5} \mathrm{H}_{4} \mathrm{R}, \mathrm{C}_{5} \mathrm{Me}_{5}$, indenilo, fluorenilo) na óptica do meu inabalável interesse pela química dos metalocenos de $\mathrm{Mo}(\mathrm{W})$ que sempre sonhei ver mais reactivos e mais fáceis de preparar.
$\mathrm{O}$ conceito subjacente era o de que a modificação dos anéis de $\mathrm{Cp}$ iria introduzir efeitos estereoquímicos e/ou electrónicos capazes de alterar significativamente a reactividade dos sistemas derivados do fragmento $\mathrm{Cp}_{2} \mathrm{Mo}(\mathrm{W})$ e torná-los cataliticamente activos.

Na realidade, este trabalho traduziu-se até agora num estudo aprofundado dos processos de escorregamento de anel ("ring-slippage" ou, de forma mais geral, deslocamentos haptotrópicos). A capacidade do anel ciclopentadienilo "escorregar" da sua habitual forma de coordenação, com os 5 átomos de carbono ligados ao metal (pentahapto coordenado, habitualmente abreviado para $\eta^{5}$ $\mathrm{Cp}$ ), para uma coordenação efectivada apenas por 3 átomos de $\mathrm{C}$ (trihapto coordenado ou $\eta^{3}-\mathrm{Cp}$ ), removendo dois electrões da camada de valência do metal, foi muitas vezes invocada desde os primórdios da química organometálica para explicar algumas observações mecanísticas. Todavia, a demonstração de que o ciclopentadienilo poderia sofrer este tipo de escorregamento sempre teve uma base experimental muito frágil: a estrutura de raios- $X$ do complexo $\left(\eta^{5}-\mathrm{Cp}\right)\left(\eta^{3}-\mathrm{Cp}\right) \mathrm{W}(\mathrm{CO})_{2}$. [10] Em contraste, 0 anel indenilo escorrega de forma muito mais expedita, $\eta^{5}$-Ind $\rightarrow \eta^{3}$-Ind, promovendo a actividade catalítica de variados complexos, nomedamente naqueles em que essa actividade envolve um mecanismo associativo ("efeito indenilo"). [11] Seria então lícito prever um aumento da reactividade dos metalocenos de Mo e W se um, ou os dois, anéis Cp fossem substituídos por indenilos. Esse trabalho, iniciado de forma sistemática em 1993 com a Tese de Doutoramento de Isabel Gonçalves, e prosseguido na Tese de Carla Gamelas e no trabalho de Beatriz Royo, produziu uma grande variedade de novos metalocenos de Mo e W (dando a volta à Big Prep!), e alargou a química dos derivados indenílicos de Mo e W, até aí quase inexistente.[12] Do conjunto de resultados obtidos, revistos em [12b], destaca-se a contribuição conseguida na interpretação do "efeito indenilo" e a constatação de que o escorregamento de anel de $\mathrm{Cp}$ pode ser fácilmente induzido nestes sistemas. As reacções descritas no Esquema 3 espelham a facilidade desses escorregamentos, nuns casos promovida por processos redox, noutros por processos de adição/remoção de ligandos.[13]

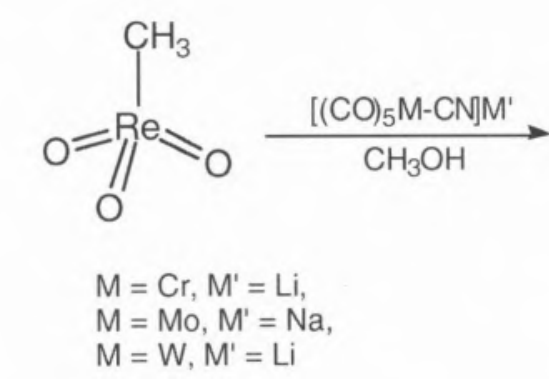

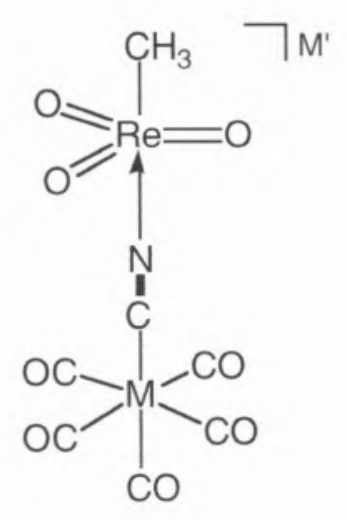

(3) 

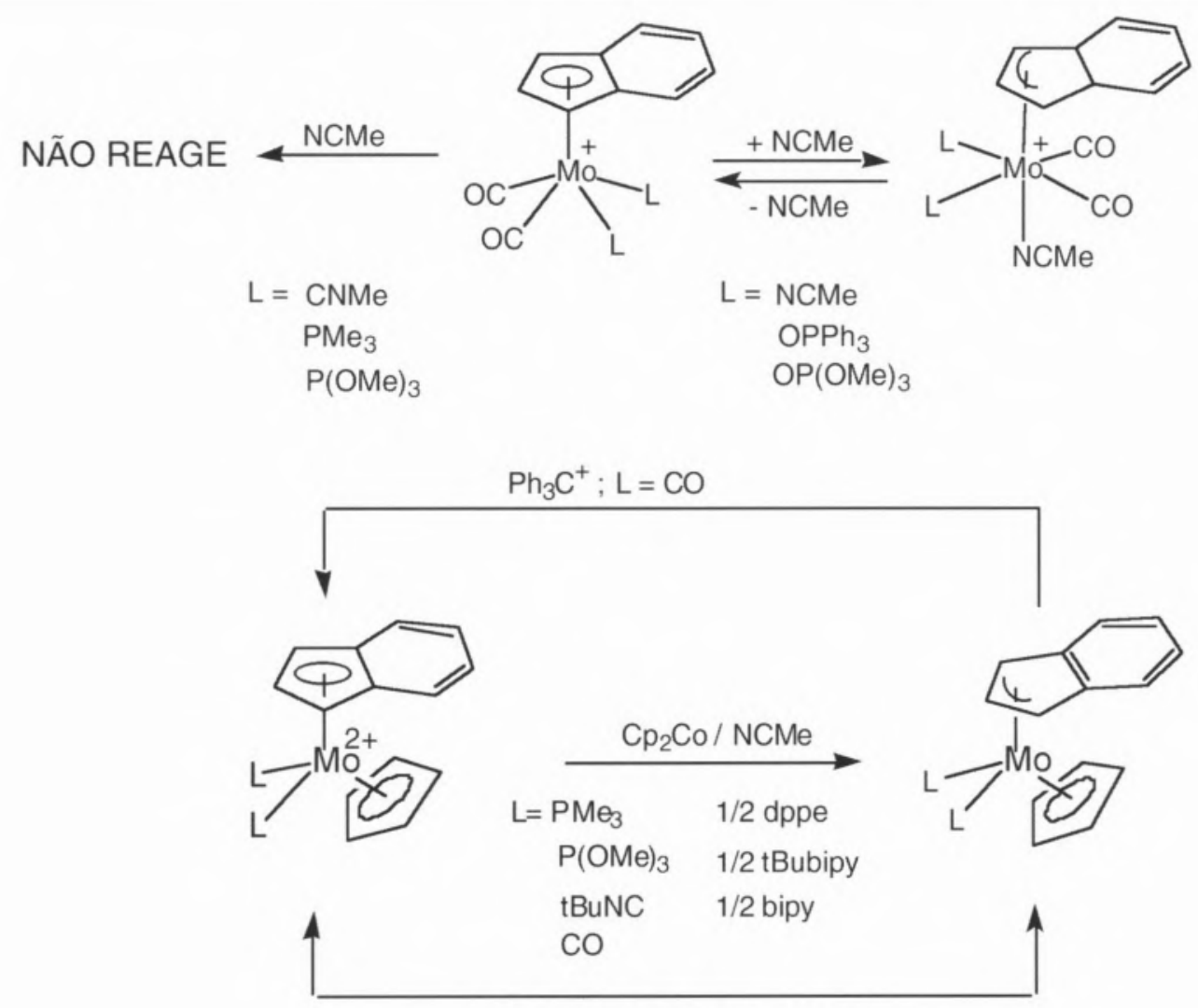

electroquimicamente reversivel

esquema 4 Escorregamento de indenilo por adiçāo de ligandos (dependente do ligando) e escorregamento de indenilo por indução redox (reversível). Esta reversibilidade existe para os $\left[\mathrm{Cp}_{2} \mathrm{ML}_{2}\right]^{2+10}$ apenas nos casos em que $\mathrm{L}=\mathrm{CO}$.

A compreensão detalhada deste fenómeno em termos da natureza da ligação química e da estrutura dos complexos intervenientes apenas foi possível graças ao recurso à Química Teórica, numa intensa colaboração com o grupo de Maria José Calhorda e com Luís Veiros (CQEIST). Entre estes estudos destaca-se a caracterização estrutural do escorregamento $\eta^{5}$-Ind $\rightarrow \eta^{3}$-Ind durante 0 processo (reversível) de redução do dicatião $\left[\left(\eta^{5}-\text { Ind }\right) \mathrm{CpMo}\left\{\mathrm{P}(\mathrm{OMe})_{3}\right\}_{2}\right]^{2+}$ a $\left[\left(\eta^{3}-\right.\right.$ Ind $\left.) \mathrm{CpMo}\left\{\mathrm{P}(\mathrm{OMe})_{3}\right\}_{2}\right][14]$ e uma reintrepretação do efeito indenilo não à luz do ganho de energia de ressonância que acompanharia a reorganização electrónica do ligando $\eta^{5}$-Ind para $\eta^{3}$ Ind (vd. Esquema 5) mas à luz da menor estabilidade dos complexos ( $\eta^{5}$-Ind) $\mathrm{ML}_{n}$ face aos análogos $\left(\eta^{5}-\mathrm{Cp}\right) \mathrm{ML}_{\mathrm{n}}$ e da estabilização dos complexos $\left(\eta^{3}-\right.$ Ind $) \mathrm{ML}_{n}$ face aos análogos $\left(\eta^{3}-C p\right) M L_{n}$. [15]

Estabelecido e caracterizado este tipo de escorregamentos resta agora tirar partido da sua existência em termos de reactividade e de aplicações catalíticas ou outras, afinal o objectivo proposto no início desta linha de trabalho. Uma maior interacção com as vertentes de aplicação industrial onde apenas me aventurei por breve mas fértil período, e uma incursão pela vertente das aplicações biológicamente relevantes (a química bio-organometálica está aí!) são os projectos que tenho em fase de arranque.

Ao contemplar retrospectivamente o caminho percorrido nestes 30 anos de Química Organometálica o que ressalta? Em primeiro lugar, a evidência do enorme progresso conseguido na investigação em Química no nosso País, incluindo a Química Organometálica e a Catálise. Em segundo lugar, a profunda satisfação de ter podido participar nessa caminhada e, de algum modo, contribuir para o seu sucesso sempre nos limites das minhas possibilidades. Por úl- timo, e para sempre, a memória de uma intensa vivência dessa "ânsia de andar para a frente" e da sua partilha com todos os colegas, supervisores e supervisionados que ao longo deste tempo se tornaram ou mantiveram meus amigos $e$ colaboradores e que, em última análise, me permitiram assinar o trabalho que descrevi. Neste contexto, o meu agradecimento especial ao Prof. Romão Dias a quem muito devo pelo entusiasmo que sempre me soube transmitir, por todo o apoio e conselho que sempre me deu e por me ter acolhido como seu "compagnon de route".

Agradecimentos

Mencionei acima alguns dos protagonistas desta história que, ou pela extensão ou pela profundidade do seu envolvimento se tornaram decisivos na obtenção dos principais resultados da minha carreira científica. A eles e a todos os outros que me abstenho de mencionar 


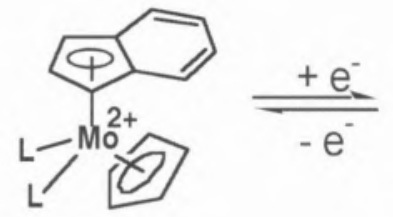

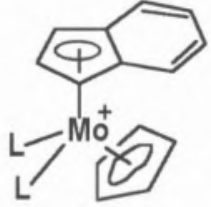

$19 \mathrm{e}$
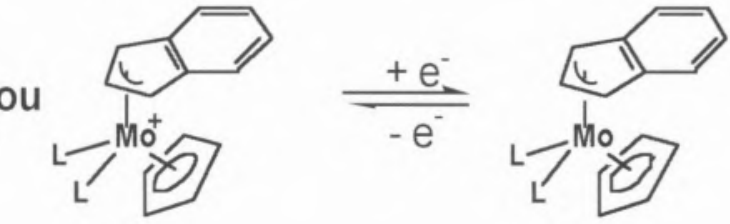

17 e

em particular, para evitar omissões injustas que seguramente ocorreriam, aqui deixo o meu mais sincero agradecimento. Num plano mais pessoal um agradecimento muito especial à João, ao Diogo e ao Vasco pelo apoio e carinho com que me ajudaram a ultrapassar os momentos menos bons e a alegria com que compartilharam os melhores.

\section{Referências}

[1] MoR $\left(\eta^{3}-C_{3} H_{5}\right)_{3}$ : Agostic Molybdenum-Alkyl Complexes. R. Benn, S. Holle, P. W. Jolly, R. Mynott and C. C. Romão, Angew. Chem. Int. Ed. Engl., 25 (1986) 555.

[2] Nucleophilic and electrophilic reactions on $\mathrm{C}_{5}$ cyclo-polyenes coordinated to the $\left[\mathrm{CpMoL}_{2}\right]^{+}$fragment $(n=1,2 ; \mathrm{L}=1 / 2 d p p e$, $\left.\mathrm{PMe}_{3}, \mathrm{P}(\mathrm{OMe})_{3}, \mathrm{CO}\right)$. C. G. de Azevedo, M. J. Calhorda, M. A. A. F. de C. T. Carrondo, A. R. Dias, A. M. Galvão, C. A. Gamelas, I. S. Gonçalves, M. F. M. da Piedade, M. T. L. Duarte and C. C. Romão, J. Organomet. Chem., 544 (1997) 257.

[3] Methylisocyanide Derivatives of Molybdenocene and Tungstenocene: Preparation, Reactivity and Electronic Structure. Crystal Structures of $\left[\left(\eta^{5}-\mathrm{C}_{5} \mathrm{H}_{5}\right)_{2} \mathrm{WBr}(\mathrm{CNMe})\right] \mathrm{Br}$ and $\left.\left[\mathrm{Cp}_{2} \mathrm{MoC} / \mathrm{N}(\mathrm{H}) \mathrm{CH}_{3}\right] \mathrm{N}(\mathrm{H}) \mathrm{C}\left(\mathrm{N}(\mathrm{H}) \mathrm{CH}_{3}\right]\right]\left[\mathrm{BF}_{4}\right]_{2}$. $\mathrm{CH}_{3} \mathrm{CN}$. M. J. Calhorda, A. R. Dias, M. T. Duarte, A. M. Martins, P. M. Matias and C. C. Romão, J. Organomet. Chem., 440 (1992) 119.

[4] Isospecific Oligo/Polymerization of Styrene with Soluble Cationic Nickel Systems. The Influence of Phosphorus Ligands. J. R. Ascenso, A. R. Dias, P. T. Gomes, C. C.
Romão, I. Tkatchenko, A. Revillon, Q.-T. Pham, Macromolecules, 29 (1996) 4172.

[5] Syntheses, Electrochemistry and Bonding of Bis(Cyclopentadienyl)Molybdenum Alkyl Complexes. Molecular Structure of $\mathrm{Mo}\left(\eta^{5}-\mathrm{C}_{5} \mathrm{H}_{5}\right)_{2}\left(\mathrm{C}_{4} \mathrm{H}_{9}\right)_{2}$. Thermochemistry of $\mathrm{Mo}\left(\eta^{5}-\mathrm{C}_{5} \mathrm{H}_{5}\right)_{2} \mathrm{R}_{2}$ and $\mathrm{Mo}\left(\eta^{5}-\mathrm{C}_{5} \mathrm{H}_{5}\right)_{2} \mathrm{~L}(\mathrm{R}=$ $\mathrm{CH}_{3}, \mathrm{C}_{2} \mathrm{H}_{5}, \mathrm{C}_{4} \mathrm{H}_{9}$ and $\mathrm{L}=$ ethylene and diphenylacetylene).M. J. Calhorda, M. A. A. F. de C. T. Carrondo, A. R. Dias, A. M. Galvão, M. H. Garcia, A. M. Martins, M. E. M. da Piedade, C. I. Pinheiro, C. C. Romão, J. A. M. Simōes and L. F. Veiros, Organometallics, 10 (1991) 483

[6] Alkyl and Arylrheniumtrioxides. W. A. Herrmann, C. C. Romão, R. W. Fischer, P. Kiprof and C. de M de Bellefon, Angew. Chem., 103 (1991) 183.

[7] Organic and Inorganic Chemistry of the $\operatorname{Re}(V I I)$ Oxides and Imido Complexes: Synthetic, Structural, Catalytic and other Studies. C. C. Romão, F. E. Kühn and W. A. Herrmann, Chem. Rev., 97 (1997) 3197

[8] Molybdenum(VI)cis-Dioxo Complexes Bearing (poly)-Pyrazolyl Methane and (poly)Pyrazolyl Borate Ligands: Synthesis, Characterization and Catalytic Applications. A. M. Santos, F. E. Kühn, I. Lucas, C. C. Romão, E. Herdtweck, J. Chem. Soc. Dalton Trans. (2001) 1332.

[9] Synthesis and Characterization of Binuclear Transition Metal-Rhenium(VII) Complexes with Bridging Cyanide Ligands. I. S. Gonçalves, F. E. Kühn, A. D. Lopes, A. J. Parola, F. Pina, J. Sotomayor and C. C. Romão, J. Organomet. Chem., 560 (1998) 117-124.

[10] The Structure of $\left(\mathrm{C}_{5} \mathrm{H}_{5}\right)_{2} \mathrm{~W}(\mathrm{CO})_{2}$, a Compound Containing a Bent trihapto-Cyclopentadienyl Ligand. G. Huttner, H. H. Brintzinger, L. G. Bell, P. Friedrich, V. Benjenke,
D. Neugebauer, J. Organomet. Chem. 145 (1978) 329

[11] The Indenyl Ligand Effect on the Rate of Substitution Reactions of Rh $\left(\eta-\mathrm{C}_{9} \mathrm{H}_{7}\right)(\mathrm{CO})_{2}$ and $\mathrm{Mn}\left(\mathrm{\eta}-\mathrm{C}_{9} \mathrm{H}_{7}\right)(\mathrm{CO})_{3}$. M. E. Rerek, L.-N. Ji, F. Basolo, J. Chem. Soc., Chem. Commun. (1983) 1208.

[12] (a) Mixed-Ring and Indenyl Analogues of Molybdenocene and Tungstenocene: Preparation and Characterization. J. R. Ascenso, C. G. de Azevedo, I. S. Gonçalves, E. Herdtweck, D. S. Moreno, M. Pessanha, and C. C. Romão, Organometallics, 14 (1995) 3901

(b) Modified Molybdenum and Tungsten Metallocenes and Ring-Slippage reactions: New Compounds and Revisited Concepts. C. C. Romāo, Appl. Organomet. Chem. 14 (2000) 539

[13] Ligand Dependence of the Indenyl Ring Slippage in $\left[\left(\eta^{5}-I n d\right) \mathrm{MoL}_{2}\left(\mathrm{CO}_{2} \mathrm{P}^{++}\right.\right.$Complexes: Experimental and Theoretical Studies. M. J. Calhorda, C. A. Gamelas, I. S. Gonçalves, E. Herdtweck, C. C. Romão and L. F. Veiros, Organometallics, 17 (1998) 2597.

[14] Stepwise Hapticity Changes in Sequential One-electron Redox Reactions of Indenyl-Molybdenum Complexes: Combined Electrochemical, ESR, X-ray and Theoretical Studies, M. E. Stoll, P. Belanzoni, M. J. Calhorda, M.

G. B. Drew, V. Félix, W. E. Geiger, C. A. Gamelas, I. S. Gonçalves, C. C. Romão, L. F. Veiros, J. Am. Chem. Soc. 123 (2001) 10595

[15] The Nature of the Indenyl Effect, M. J. Calhorda, Carlos C. Romão, L. F. Veiros, Chem. Eur. J. no prelo (2001). 